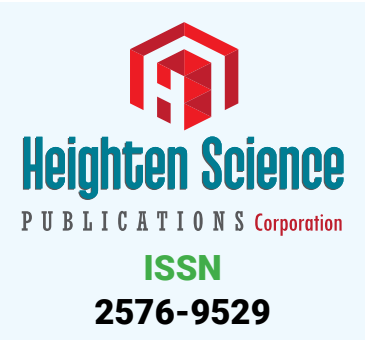

*Address for Correspondence: Nasrulla Abutaleb, Diaverum: PADC, Jeddah, Saudi Arabia, Tel: +996555698247; Email:

abutaleb_k@hotmail.com

Submitted: 18 June 2019

Approved: 26 June 2019

Published: 27 June 2019

Copyright: ( 2019 Abutaleb N. This is an open access article distributed under the Creative Commons Attribution License, which permits unrestricted use, distribution, and reproduction in any medium, provided the original work is properly cited

Check for updates
Research Article

\section{Alternate day outpatient hemodialysis schedule is the appropriate practical alternative schedule to improve patients' outcomes}

\author{
Nasrulla Abutaleb* \\ Diaverum: PADC, Jeddah, Saudi Arabia
}

\section{Introduction}

The rise in all-cause and cardiovascular mortality and hospitalization rates among the hemodialysis (HD) patients after the long weekly inter-dialysis interval is very impressive. In fact, there is an additional long-term morbidity risk besides this acute rise in mortality and hospitalizations that can be expected from the weakly exaggerated pre-dialysis peaks of the less risky hemodynamic and biochemical parameters. An approach for eliminating these long weekend inter-dialysis intervals is through the provision of the hemodialysis sessions on every other day (EOD) basis, regardless of the week days. This is likely to be both practical and cost-effective. Such EOD schedule can be introduced easily beside the ongoing thrice weekly HD schedule without disturbance of the HD unit work. The availability of EOD schedule would provide a healthier and cost-effective alternative schedule for those patients who can't tolerate the weekend intervals and for those looking for maintaining their long-term health on the option of hemodialysis. In fact, with available data, medical staff is expected to encourage all patients to shift their HD to the EOD schedule.

\section{Why we need an alternative HD Scheduling}

Significant rise of the mortality and acute morbidity rates (M \& M) after the long weekly inter-dialysis interval has been well documented by multiple studies [1-3]. The rise in all-cause and cardiovascular mortality and hospitalization rates after these weekend intervals is very impressive and should not ideally be left unaddressed. The figures reported for the rise in all-cause mortality ranged from $23 \%$ [1], to $41 \%$ [2]. The rise in the rates of cardiac mortality and hospitalization after the weekend interval is even more pronounced; RN Foley et al., reported $36 \%(\mathrm{P}=0.007)$ and $124 \%$ rise in cardiac mortality and hospital admission for cardiovascular event (CVE), respectively [1]. T Springel et al., reported an admission rate after the weekend interval of 2.6 times the rate in the rest of the week days [3]. An almost similar degree to above hospitalization rate of 2.6 times is the figure of $240 \%$ rise in CVE rate after the long inter-dialysis interval that was reported by J. E Hakmei et al. [4]. These authors reported a rise of $260 \%$ in the CVE rate after 3 days gap that can be seen with unusual schedules as that of Mon-Wed-Thu or Tue-Wed-Fri. These hospital admissions may not be that selflimited; the reported high rate of rehospitalization among HD patients is high. Renal Data System has revealed an overall rehospitalization rate of 34\% within 30 days of discharge [5]. This finding suggests a high rate of dragging morbidity after such initial 
hospitalizations. It is worthy to indicate that the Australian and New Zealand Dialysis and Transplant (ANZDATA) Registry data has revealed the absence of such rise among patients receiving more than thrice-weekly hemodialysis sessions [6]. In fact, earlier studies on frequent daily Hemodialysis has revealed gains in both patients' survival and in regression of the Left Ventricle hypertrophy benefit, LVH.

The harm from accumulating toxins or from the hemodynamic stress is generally related to both the peak levels and the overall exposure or the AUC (area under the curve); both of which are increased significantly by the long weekend inter-dialysis gap. This may be said about the potential harms of hyperphosphatemia, metabolic acidosis, subclinical overload, higher BP and arterial stiffness in addition to the higher rates of organ ischemia when higher UF rate is utilized on HD session following the weekend. The interdialytic increase in central wave augmentation index was documented by $\mathrm{P}$. I. Georgianos et al to be more pronounced during the 3-day interval; this index reflects the degree of arterial stiffness and has been associated with increased cardiovascular risk [7]. Subtle echocardiographic dysfunction after the weekend intervals have also been documented by M. Obokata [8]. As alluded to above, the weekly pathological peaks of these parameters are not expected to result directly into above stated acute rise in $M \& M$ associated with the weekend intervals bur are of course expected to account for the currently high long-term M \&M. We can see that EOD regimen would improve the standard KT/V significantly by reducing the average pre-dialysis peaks significantly. In fact, many of the benefits attributed to quotidian HD schedule are justifiably well expected to be maintained on adopting the more practical schedule of EOD hemodialysis.

\section{Medical non-compliance}

Compared with compliant patients, those who skipped one or more HD sessions in a month had a $25 \%$ higher risk of death $(\mathrm{P}<0.01)$ [9]. Higher figure of $33 \%$ for the mortality associated with skipping one HD session or more per month was reported also from DOPPS data [10]. Missing a midweek HD session would normally result in a 3-days gap between the dialysis days; the associated reported rise of CVE rate was $260 \%$ which was not very different from the comparable figure of $240 \%$ after the usual 2-days weekend gap.

We need to pause! Extending the inter-dialysis gap from one to three days by the patient on missing a midweek HD session is considered a risky irresponsible behavior while extending that gap from one to two days on weekly basis by the medical authorities is an acceptable policy despite above stated terrifying rises on M \& M rates besides the additional long-term negative health consequences. I believe, we have to admit that our strict adherence to the thrice weekly HD schedule is another form of non-compliance with available facts. It is something that needs to be rectified if we are looking for further improvement of patients' survival on HD.

Offering monthly rather than weekly based HD schedule for the compliant patients

It is unavoidable to have such long inter-dialysis intervals as long as the HD sessions are arranged on a weekly basis. Earlier suggestions to address the problem of long inter-dialysis interval were not practical because of the intrinsic limitations imposed by looking for a solution within the "week box". Such suggestions included the provision of a $4^{\text {th }}$ weekly HD session on either Saturdays or Mondays (The days before and after Sundays). Such approach is not practical because of the limited capacity in the staff and available HD slots in most of HD units which would not allow accommodating all patients together every Saturday and Monday. Doubling the number of HD staff 
and slots will then be needed on Saturdays and Mondays. The same inefficiency can be said about the other suggestions of short daily HD and long nocturnal in-center HD approaches. Home hemodialysis is a true alternative approach though its acceptance is still limited to a small proportion of the HD population.

I believe we need to liberate our in-center HD schedules from being stuck with those weekly schedules. The EOD approach, odd vs. even days of each month, regardless of the week days constitutes a simple, practical cost effective solution to eliminate the long inter-dialysis interval. The HD schedule will be based on monthly rather than weekly basis. Such schedule can be offered for the compliant HD patients who are looking for better health on chronic HD and for those in need for frequent ( $>$ three sessions/week) HD sessions. The old thrice weekly schedule can continue undisturbed in the same HD units for the rest of the patients in parallel with the monthly based schedule. Simply, every two patients agreeing to adopt EOD dialysis will take each other dialysis slots every other week. i.e. each patient will receive hemodialysis every other Sunday and will assume Tuesday-Thursday-Saturday in that week then MondayWednesday- Friday in the following week. Such alternating schedule would not disturb that of the remaining patients on the usual thrice weekly schedule. Patients on EOD schedule would need just to stick to HD on every other day (either odd or even days of each month) regardless of the week days. In fact, assuming that we have two daily HD shifts and accepting a nurse: patient ratio of 1 to 3 , we can put 40 patients on such EOD schedule for every three 3 nurses (beside an on-call physician) agreeing to work on Sundays. Such three nurses working on Sundays can dialyze 20 patients every Sunday allowing a total of 40 patients to be put on EOD schedule. With appropriate explanation, the dialysis medical staff is likely to accept such approach as is already being practiced by almost all other medical staff who can't interrupt their service because of the weekends; it would eliminate one major defect in the validity of hemodialysis as an RRT modality. The dialysis medical staff has already scarified half of their weekends by working on Saturdays which was a half solution for the problem of long weekend inter-dialysis interval; but the current 2-days weekend gap is still unsafe and needs to be addressed. Yielding to medical facts, I would suggest starting a process of gradual shifting of the current HD schedule toward EOD.

\section{References}

1. Foley RN, Gilbertson DT, Murray T. Long Interdialytic Interval and Mortality among Patients Receiving Hemodialysis. N Engl J Med. 2011; 365: 1099-1107. Ref.: https://bit.ly/2NfLiH9

2. Zhang $H$, Schaubel DE, Kalbfleisch JD. Dialysis outcomes and analysis of practice patterns suggests the dialysis schedule affects day-of-week mortality. Kidney Int. 2012; 81: 1108-1115. Ref.: https://bit.ly/2IR7LpE

3. Springel T, Laskin B, Shult J. Longer interdialytic interval and cause-specific hospitalization in children receiving chronic dialysis. Nephrol Dial Transplant. 2013; 28: 2628-2636. Ref.: https://bit.ly/2NcLBSW

4. Hakmei EJ, Nietert PJ, Fitzgibbon WR. Length of Interdialytic Intervals Affects Morbidity and Mortality in Chronic Haemodialysis Patients. J Clin Exp Nephrol. 2017: 2: 38. Ref.: https://bit.ly/2Nhxegn

5. US Renal Data System. USRDS 2013 Annual Data Report: Atlas of Chronic Kidney Disease \& EndStage Renal Disease in the United States. Bethesda, MD, National Institutes of Health. National Institute of Diabetes and Digestive and Kidney Diseases. 2013.

6. Georgianos PI, Sarafidis PA, Sinha AD. Adverse Effects of Conventional Thrice-Weekly Hemodialysis: Is It Time to Avoid 3-Day Interdialytic Intervals? Am J Nephrol. 2015; 41: 400-408. Ref.: https://bit.ly/2IQOmW0

7. Georgianos PI, Sarafidis PA, Haidich AB. Diverse effects of interdialytic intervals on central wave augmentation in hemodialysis patients. Nephrol Dial Transplant. 2013; 28: 2160-2169. Ref.: https://bit.ly/2NfNf6r

8. Obokata M, Negishi K, Marwick TH. Comparison of different interdialytic intervals among 
hemodialysis patients on their echocardiogram-based cardiovascular parameters, American Heart Journal. 2015; 169: 523-530. Ref.: https://bit.ly/2xg1PQE

9. Leggat JE, Orzol SM, Hulbert-Shearon TE, Golper TA, Jones CA. Noncompliance in hemodialysis: Predictors and survival analysis. AJKD. 1998; 32: 139-145. Ref.: https://bit.ly/2RLQwJr

10. Saran R, Bragg-Gresham JL, Rayner HC. Nonadherence in hemodialysis: Associations with mortality, hospitalization, and practice patterns in the DOPPS. Kidney International. 2003; 64: 254-262. Ref.: https://bit.ly/2KHaPqo 